\title{
Escherichia coli superoxide dismutase expression does not change in response to iron challenge during lag phase: Is the ferric uptake regulator to blame?
}

\author{
Robert L. Bertrand*, Michael O. Eze \\ Health Enhancement Biochemistry Laboratory, Department of Chemistry, University of Winnipeg, Winnipeg, Canada; \\ \#Corresponding Author: m.eze@uwinnipeg.ca
}

Received 19 July 2013; revised 23 August 2013; accepted 10 September 2013

Copyright (C) 2013 Robert L. Bertrand, Michael O. Eze. This is an open access article distributed under the Creative Commons Attribution License, which permits unrestricted use, distribution, and reproduction in any medium, provided the original work is properly cited.

\begin{abstract}
Intracellular iron levels and the expression of superoxide dismutase (SOD) and hydroperoxidase (HP) are regulated in Gram-negative bacteria by the iron(II)-activated ferric uptake regulator (Fur). We have previously observed that the expression of SOD in exponential phase Escherichia coli is dependent upon the redox state of iron in media, consistent with the ferrous specificity of Fur regulation (Bertrand et al., Med. Hypotheses 78: 130 - 133, 2012). Through the non-denaturing electrophoretic technique we have determined the Escherichia coli expression profiles of SOD and HP in response to iron challenge throughout lag, logarithmic, and stationary phases of replication. Lag phase SOD presented an unusual expression profile such that SOD expression was unresponsive to iron challenge, analogous to observations of mutant strains lacking Fur and of $E$. coli incubated in iron-deplete media. Challenging Escherichia coli with iron during logarithmic phase revealed that length of exposure to oxidants is unlikely to be the cause of SOD unresponsiveness in lag phase. HP activity was up-regulated two- or three-fold throughout all growth phases in response to iron challenge, but did not present redox- or growth phase-specific outcomes in a manner analogous to SOD. We hypothesize that low Fur levels during lag phase are responsible for unresponsive SOD.
\end{abstract}

Keywords: Antioxidant Enzymes; Oxidative Stress;

*Present Address: Department of Chemistry, University of Manitoba, Winnipeg, Canada.
Iron Metabolism; Enzyme Expression

\section{INTRODUCTION}

Iron is an essential metal, required as a prosthetic group for many metallo-proteins. However, iron potentiates oxidative stress through the catalytic formation of hydroxyl radicals from hydrogen peroxide through the Fenton reaction [1] (Eq.1):

$$
\mathrm{Fe}^{2+}+\mathrm{H}_{2} \mathrm{O}_{2} \rightarrow \mathrm{Fe}^{3+}+\mathrm{OH} \cdot+\mathrm{OH}^{-}
$$

Reactive oxygen species such as hydroxyl radical $(\mathrm{OH} \cdot)$, superoxide $\left(\mathrm{O}_{2}^{-{ }^{-}}\right)$, and hydrogen peroxide $\left(\mathrm{H}_{2} \mathrm{O}_{2}\right)$ have been implicated in numerous pathologies as well as the process of aging [2-8]. Cells must therefore carefully regulate intracellular iron levels to meet metabolic needs yet mitigate iron cytotoxicity [9-12]. In Gram-negative bacteria such as Escherichia coli, this balance is mediated by the ferric uptake regulator (Fur), an iron (II)dependent suppressor of iron uptake proteins [13-15]. As Fur also regulates superoxide dismutase (SOD) and hydroperoxidase (HP) [16-23], two essential antioxidant enzymes for respiring bacteria [24,25], Fur is de facto a homeostatic mediator of intracellular oxidative conditions.

SOD isozymes are distinguished and named by their metal prosthetic groups. Escherichia coli, the model species of this study, copiously produces manganese- and iron-bearing forms (MnSOD, FeSOD), as well as a trace isozyme known as CuZnSOD [26,27]. These are encoded by the genes $\operatorname{sodA}$, sodB, and $\operatorname{sod} C$, respectively. Unlike FeSOD, MnSOD expression levels vary greatly in response to a variety of oxidative stimuli $[28,29]$. MnSOD expression is regulated by the protein products of six global regulators: soxRS and soxQ, both of which activate sodA; and fur, arcA, fnr, and ihf, all of which sup- 
press sodA [17,29]. E. coli produces two isozymes of hydroperoxidase: HPI, encoded by katG, and HPII, encoded by katE $[30,31]$. HPI is the principle responder to oxidative threats, and its expression is activated by the $\mathrm{H}_{2} \mathrm{O}_{2}$-inducible OxyR regulon [32]. HPII is predominantly active during stationary phase and serves as part of the starvation response controlled by the rpoS regulon [32-36]. The rpoS regulon has also been observed to control HPI expression to some extent [35,37]. Combined, these antioxidant enzymes eliminate superoxide and hydrogen peroxide in the following pair of reactions (Eqs.2,3):

$$
\begin{aligned}
& \mathrm{O}_{2}{ }^{--}+2 \mathrm{H}^{+} \stackrel{\mathrm{SOD}}{\longrightarrow} \mathrm{H}_{2} \mathrm{O}_{2} \\
& 2 \mathrm{H}_{2} \mathrm{O}_{2} \stackrel{\mathrm{HP}}{\longrightarrow} 2 \mathrm{H}_{2} \mathrm{O}+\mathrm{O}_{2}
\end{aligned}
$$

Sodium nitroprusside (SNP) is an iron(II)-bearing penta-cyanide and a potential therapeutic agent for acute cardiopulmonary emergencies [38-42]. SNP rapidly releases nitric oxide (NO) in vivo by forming S-nitrosothiols with sulfhydryl-containing compounds such as glutathione, transporting $\mathrm{NO}$ throughout the vasculature [43]. The utility of SNP in the present study is threefold as it allows examination of the biochemistry of both iron and nitric oxide, and may provide insights relevant to therapeutics. Nitric oxide, itself an oxidant, is an endogenous regulator of multiple metabolic pathways and an inhibitor of iron-bound Fur [44-46]. We have previously observed that logarithmic-phase E. coli treated with SNP lowered expression of MnSOD, but the expression levels of FeSOD were greatly enhanced [47]. As NO activates MnSOD transcription [48,49], and as FeSOD expression is generally unresponsive to oxidative stimuli $[28,29]$, it was predicted beforehand that treatment of E. coli with SNP should increase MnSOD expression and decrease FeSOD expression. That we had observed the opposite was a most unexpected finding [47]. Among the six regulators of MnSOD, Fur is uniquely known, upon activation by iron, to both inhibit MnSOD transcription and by post-transcriptional means de-suppress FeSOD [13,16,17,20,50,51]. We therefore suggested SNP be activating Fur, the implications of this hypothesis on human health and disease having already been discussed [47]. Supporting this hypothesis were the comparative effects of potassium ferricyanide (PFi) and potassium ferrocyanide (PFo) on E. coli, both of which are structural analogs of SNP that cannot release NO. Though incubating E. coli in the presence of the ferric PFi had unremarkable effects on SOD expression, treatment with ferrous PFo mimicked SNP-associated expression patterns consistent with the ferrous specificity of Fur activation [47]. These results were obtained through activity staining assays of $E$. coli extracts run through non-denaturing electrophoresis.
We adopted this successful methodology to elucidate the expression profiles of both superoxide dismutase (SOD) and hydroperoxidase (HP) throughout all phases of $E$. coli growth under aerobic conditions using SNP as well as the ferric and ferrous analog standards as oxidative challengers. The objective of this study is to characterize antioxidant enzyme response to iron challenge within the context of oxidative stress, and to identify plausible regulatory elements underlying antioxidant enzyme response patterns. Herein we have determined that all three iron treatments failed to change the expression of either isozyme of SOD during lag phase of E. coli replication. MnSOD expression was markedly higher during lag phase. We hypothesize that low Fur activity during lag phase is the cause of the recalcitrant SOD as well as heightened MnSOD expression. Indirect evidence supporting this hypothesis is provided in other enzyme studies, which will be discussed. HP was responsive to iron treatments during all phases, but no redox- or phasespecific behaviour analogous to that of SOD was observed.

\section{METHODS}

\subsection{Culture Conditions}

Escherichia coli (ATCC 8677) cultures, originating from $1 \%$ inocula of overnight cultures, were incubated aerobically at $37^{\circ} \mathrm{C}$ on shaker bath (75 RPM) in pre-warmed $0.2 \%$ glucose-based M9 liquid media (500 $\mathrm{mL}$ in $1000 \mathrm{~mL}$ Erlenmeyer flasks) with sterile cotton stoppers (Overnight cultures were similarly incubated in $500 \mathrm{~mL}$ of M9 media). Media contained $1.00 \mathrm{mM}$ SNP, $\mathrm{PFo}$, or $\mathrm{PFi}$, introduced aseptically either at the start of incubation or after five hours of incubation. Growth rates were monitored by periodic optical density readings at $550 \mathrm{~nm}$. The E. coli strain employed was chosen simply because it was available for research.

\subsection{Protein Preparation and Gel Electrophoresis}

After 1, 6, or $24 \mathrm{~h}$ of incubation, E. coli cells were harvested by centrifugation, and the supernatant replaced with a $100 \mathrm{mM}$ HEPES buffer solution (pH 7.3) containing $1 \mathrm{mM} \mathrm{MgCl}_{2}$ and protease inhibitor cocktail (Sigma). Cells were lysed by abrasion vortexing, and the cellular debris removed by centrifugation. Total protein concentration was determined by the method of Bradford [52], and the crude extracts were diluted to $0.6 \mathrm{mg} / \mathrm{ml}$ total protein. These dilutions permitted quantitation of the relative differences in the expression of SOD and HP between treated and untreated cultures following electrophoresis and activity assays. $20 \mu \mathrm{L}$ of each extract were loaded into $7.5 \%$ non-denaturing polyacrylamide 
gels (29:1), and electrophoresed at $200 \mathrm{~V}$ and $0.03 \mathrm{~A}$ for approximately two hours using a TRIS-glycine running buffer. At least three experiments were conducted per treatment and growth phase.

\subsection{Enzyme Activity Assays}

SOD activity was determined by the method of Beauchamp and Fridovich [53], with modifications as described by Britigan and colleagues [54]: A $50 \mathrm{mM}$ phosphate buffer solution ( $\mathrm{pH}$ 7.8) containing $0.25 \mathrm{mM}$ nitroblue tetrazolium (NBT), $1 \mathrm{mM}$ ethylenediaminetetraacetate (EDTA), $28 \mathrm{mM}$ tetramethylethylenediamine (TEMED), and $0.03 \mathrm{mM}$ riboflavin was applied to gels in darkness for 45 minutes, stirring occasionally, followed by destaining in darkness with phosphate buffer for 45 minutes. Achromatic bands appear on a blue-purple background upon exposure to light. HP activity was assayed by the method of Woodbury and colleagues [55]: Gels were immersed in a $0.003 \%(w / v) \mathrm{H}_{2} \mathrm{O}_{2}$ solution for ten minutes, followed by rinsing and addition of a freshly-prepared solution of $2 \%(\mathrm{w} / \mathrm{v})$ potassium ferricyanide and $2 \%(\mathrm{w} / \mathrm{v})$ ferric chloride for five minutes. Achromatic bands appear on a dark green background. Band intensities were quantified with a band analyzer (Vakili Gel Analysis Unit, Dept. of Chem., U. of Winnipeg).

\subsection{Enzyme Activity Ratios}

To determine how much SOD isozyme concentrations changed in E. coli in response to treatment, enzyme activity ratios were generated by dividing the determined isozyme concentration in treated samples by the concentration in the corresponding untreated standard. For example, if MnSOD from SNP-treated E. coli during exponential phase was half as concentrated as MnSOD from untreated $E$. coli during the same phase, an enzyme activity ratio of 0.50 would be reported. Isozyme concentrations are the average determination of a minimum of three independent experiments. Data from unpublished work was included in the data sets (when appropriate) to provide as many as 10 independent experiments in some instances. The average was five experiments. Data points exceeding the lower and upper quartiles by 1.5 times were removed as outliers. The enzyme concentrations were approximated by comparing band intensity values between sample bands and that of a known quantity of a standard enzyme loaded into gels. These standard enzymes were $10 \mathrm{ng}$ of Corynebacterium glutamicum catalase (Fluka) and $50 \mathrm{ng}$ of E. coli manganese SOD (Sigma). Ratio significance was determined through Student's T-test and pooled standard deviation at 90 and 95 percent confidence.

\section{RESULTS}

\subsection{E. coli Growth Curves}

Untreated $E$. coli remained in lag phase for approximately two hours, following which cultures progressed into an exponential phase that ended after eight additional hours of incubation (Figure 1). $1.00 \mathrm{mM} \mathrm{SNP}$ was observed to uniquely hinder E. coli replication, evident by mid-exponential phase, resulting in a lower optical density at stationary plateau as compared to all other treatments (Figure 1). These results suggest that SNP imparts a severe oxidative and/or metabolic threat to $E$. coli.

\subsection{E. coli Growth Curves}

E. coli cells were incubated in the presence of 1.00 mM SNP, PFo, or PFi, and harvested after 1, 6, or 24 hours of incubation in accordance with the middle of its determined lag, exponential, and stationary periods (Figure 1). SOD assays of electrophoresed extracts and SOD activity ratios are provided (Figure 2; Table 1). Unlike exponential and stationary-phase harvests (Figures 2(b) and (c)), no treatment was capable of changing SOD isoyzme activities during lag phase, as the activity ratio was approximately 1 and did not deviate significantly from the untreated standard (Table 1). There are at least two possible reasons why this occurred.

It is possible that: 1) induction of SOD is time-dependent, and one hour is not enough time to observe antioxidant adaptation to oxidative challenge; or that 2) there are underlying regulatory reasons why iron challenge is unable to change SOD activities during lag

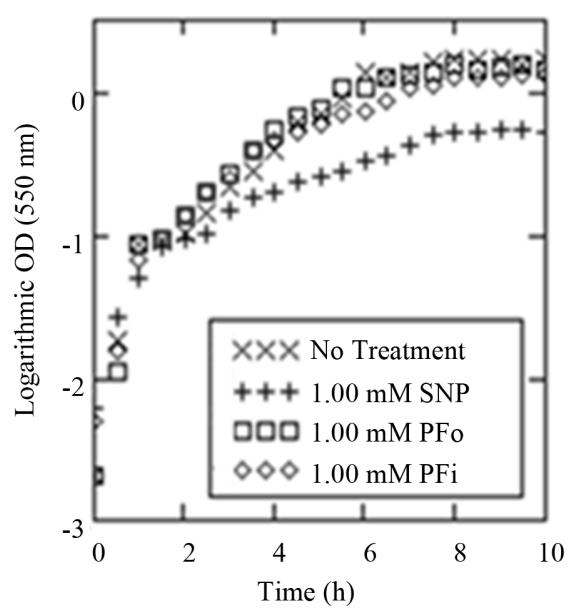

Figure 1. Growth curves of E. coli treated with $1.00 \mathrm{mM}$ sodium nitroprusside (SNP), $1.00 \mathrm{mM}$ potassium ferrocyanide ( $\mathrm{PFo}$ ), or $1.00 \mathrm{mM}$ potassium ferricyanide (PFi), incubated as described (see "Methods"). Growth curve is representative of at least three independent trials. 

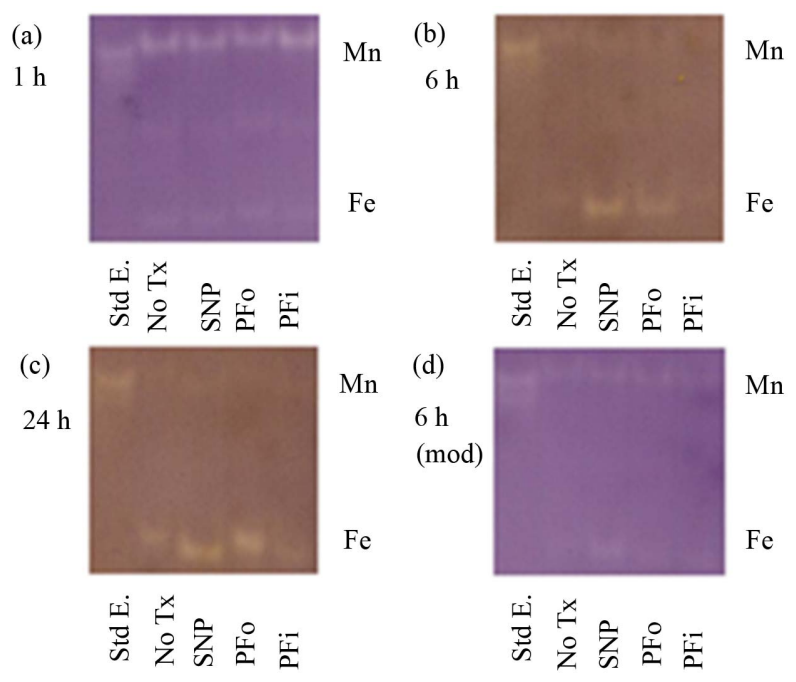

Figure 2. E. coli SOD expression profiles, presented as activity bands on non-denaturing polyacrylamide gels. E. coli originating from overnight cultures were incubated as described (see "Methods") in the presence of $1.00 \mathrm{mM}$ sodium nitroprusside (SNP), $1.00 \mathrm{mM}$ potassium ferrocyanide (PFo), or $1.00 \mathrm{mM}$ potassium ferricyanide (PFi), then harvested after 1, 6, or $24 \mathrm{~h}$ of incubation (a-c). " $6 \mathrm{~h}$ (mod)" are E. coli harvested after $6 \mathrm{~h}$ of incubation, but only received $1 \mathrm{~h}$ of treatment (addition at 5 h) (d). $20 \mu \mathrm{L}$ of $0.6 \mathrm{mg} / \mathrm{ml}$ crude protein extract were loaded into each well, and electrophoresed and stained for SOD activity as described (see "Methods"). "Std E." is $50 \mathrm{ng}$ of E. coli MnSOD, used as a positive standard. "No Tx" is untreated $E$. coli, equivalently harvested. Under present conditions, E. coli MnSOD is electrophoretically slowest (top band) and FeSOD fastest (bottom band) [71,72]. As the staining technique is light-activated, and gel backgrounds darken with more light exposure, variations in gel background colour from trial to trial will inevitably arise. Gels are representative of at least three independent trials.

phase. To investigate, liquid broths of $E$. coli were equivalently incubated without treatment for five hours, following which $1.00 \mathrm{mM}$ (final concentration) of SNP, $\mathrm{PFo}$, or PFi was added to each broth. These cultures were incubated for $1 \mathrm{~h}$ more, and then harvested. If SOD is unresponsive to iron challenge because $1 \mathrm{~h}$ of incubation is not enough time for E. coli to change SOD expression levels, then SOD should remain similarly unchanged when $E$. coli cells are exposed to the same iron challenge for $1 \mathrm{~h}$ during exponential phase. If, however, SOD is unresponsive to iron challenge because of underlying regulatory reasons determining SOD activity during lag phase, then $1 \mathrm{~h}$ exposure to iron challenge during exponential phase should result in a different expression profile than that observed in lag phase. Such "modified" exponential phase trials would likely be similar to other exponential phase trials with $6 \mathrm{~h}$ of exposure to treatments.

This predicted outcome was indeed observed, albeit with some statistical caution (Figure 2(d)). SNP and PFo
Table 1. Activity ratios of E. coli SOD isozymes throughout all growth phases under treatment of $1.00 \mathrm{mM} \mathrm{SNP,} \mathrm{PFo,} \mathrm{or} \mathrm{PFi.}$

\begin{tabular}{|c|c|c|c|c|c|}
\hline \multirow{2}{*}{ Growth Phase } & \multirow{2}{*}{ Treatment } & \multicolumn{2}{|c|}{ MnSOD } & \multicolumn{2}{|c|}{ FeSOD } \\
\hline & & Ratio & SD & Ratio & SD \\
\hline \multirow{3}{*}{ Lag phase $(1 \mathrm{~h})$} & SNP & \multicolumn{2}{|c|}{$1.07 \pm 0.98$} & \multicolumn{2}{|c|}{$0.92 \pm 0.55$} \\
\hline & PFo & \multicolumn{2}{|c|}{$0.80 \pm 0.52$} & \multicolumn{2}{|c|}{$1.20 \pm 0.49$} \\
\hline & $\mathrm{PFi}$ & \multicolumn{2}{|c|}{$0.81 \pm 0.43$} & \multicolumn{2}{|c|}{$1.04 \pm 0.50$} \\
\hline \multirow{3}{*}{$\begin{array}{l}\text { Exponential } \\
\text { phase }(6 \mathrm{~h})\end{array}$} & SNP & \multicolumn{2}{|c|}{$0.76 \pm 0.64$} & \multicolumn{2}{|c|}{$3.16 \pm 3.15^{*}$} \\
\hline & PFo & \multicolumn{2}{|c|}{$0.50 \pm 0.43^{*}$} & \multicolumn{2}{|c|}{$1.51 \pm 1.75$} \\
\hline & $\mathrm{PFi}$ & \multicolumn{2}{|c|}{$0.80 \pm 0.60$} & \multicolumn{2}{|c|}{$0.60 \pm 0.72$} \\
\hline \multirow{3}{*}{$\begin{array}{c}\text { Stationary } \\
\text { phase }(24 \text { h) }\end{array}$} & SNP & \multicolumn{2}{|c|}{$1.59 \pm 1.94$} & \multicolumn{2}{|c|}{$2.63 \pm 1.05^{*}$} \\
\hline & PFo & \multicolumn{2}{|c|}{$0.55 \pm 0.52^{* *}$} & \multicolumn{2}{|c|}{$1.93 \pm 1.01$} \\
\hline & $\mathrm{PFi}$ & \multicolumn{2}{|c|}{$0.48 \pm 0.48^{*}$} & \multicolumn{2}{|c|}{$1.39 \pm 0.65$} \\
\hline \multirow{3}{*}{$\begin{array}{l}\text { "Modified" } \\
\text { Exponential } \\
\text { phase }(6 \mathrm{~h})\end{array}$} & SNP & \multicolumn{2}{|c|}{$0.66 \pm 0.58$} & \multicolumn{2}{|c|}{$2.58 \pm 2.02^{* *}$} \\
\hline & PFo & \multicolumn{2}{|c|}{$0.56 \pm 0.51$} & \multicolumn{2}{|c|}{$1.61 \pm 1.21$} \\
\hline & PFi & \multicolumn{2}{|c|}{$0.96 \pm 0.87$} & \multicolumn{2}{|c|}{$1.01 \pm 0.73$} \\
\hline
\end{tabular}

"Ratio" is defined as the average SOD isozyme concentration of the treated $E$. coli divided by the average SOD isozyme concentration of untreated $E$. coli under identical conditions. Significance at $90\left(^{*}\right)$ and $95\left(^{* *}\right)$ percent confidence was determined through Student's T-test and pooled standard deviation.

both reduced MnSOD activity by about one-third as compared to untreated $E$. coli, but were only significant at $\mathrm{P} \leq 0.36$ and $\mathrm{P} \leq 0.25$, respectively, suggesting there is an approximate one third to one quarter chance that deviation was caused by random error alone. PFi failed to change MnSOD, with an approximate 94 percent chance of variance being due to random error. FeSOD expression was heightened by SNP and PFo treatment 2.58 and 1.61 fold, respectively, but only SNP treatment reached statistical reliability at $\mathrm{P} \leq 0.05$ (Table 1).

We therefore conclude that the hypothesis that there are underlying regulatory reasons distinct to lag phase causing SOD unresponsiveness is more likely correct. It is remarkable that PFi was also capable of decreasing MnSOD activity alongside PFo during stationary phase (Table 1). It is plausible that this may be caused by redox shifts in media, changes in intracellular iron import and export rates, changes in iron solubility influencing Fur activation, or other causes. Strangely, SNP induced a 50 percent increase in MnSOD activity at this time; however, deviation is likely due to random error as statistical analysis shows an approximate 90 percent chance of being caused by random error.

Extracts of untreated E. coli harvested from all three growth phases were electrophoresed and stained for SOD activity. MnSOD was found to display remarkably higher activity during lag phase (Figure 3). In summary, SOD assays show markedly higher MnSOD expression during lag phase. This heightened expression is immutable to iron treatment, and SOD recalcitrance does not seem to 
be caused merely by inadequate time exposure to iron challengers.

\subsection{Hydroperoxidase}

The expression profiles of HP isozymes from E. coli treated with $\mathrm{SNP}, \mathrm{PFi}$, and PFo were determined in the same manner as that of SOD. Activity ratios for HP isozymes are provided (Table 2). E. coli exposed to any treatment responded with heightened HP activity during all phases of replication, albeit with varying levels of statistical certainty per treatment and growth phase (Figure 4; Table 2). Treatments during lag phase raised HP expression by 50 to 150 percent, but failed to reach

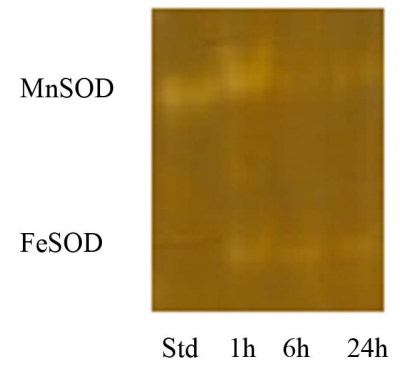

Figure 3. Electrophoresis and SOD staining of untreated $E$. coli extracts, harvested after ( $\mathrm{L}$ to $\mathrm{R}$ ) 1, 6, and $24 \mathrm{~h}$ of incubation, as described (see "Methods"). "Std" is $50 \mathrm{ng}$ of E. coli MnSOD. Gel is representative of at least three trials.

Table 2. Activity ratios of E. coli HP isozymes throughout all growth phases under treatment of $1.00 \mathrm{mM} \mathrm{SNP,} \mathrm{PFo,} \mathrm{or} \mathrm{PFi.}$

\begin{tabular}{|c|c|c|c|c|c|}
\hline \multirow{2}{*}{ Growth Phase } & \multirow{2}{*}{ Treatment } & \multicolumn{2}{|c|}{ HPII } & \multicolumn{2}{|c|}{ HPI } \\
\hline & & Ratio & SD & Ratio & SD \\
\hline \multirow{3}{*}{ Lag phase (1 h) } & SNP & \multicolumn{2}{|c|}{$1.64 \pm 1.52$} & \multicolumn{2}{|c|}{$2.32 \pm 2.35$} \\
\hline & PFo & \multicolumn{2}{|c|}{$1.50 \pm 1.76$} & \multicolumn{2}{|c|}{$1.54 \pm 1.71$} \\
\hline & PFi & \multicolumn{2}{|c|}{$1.64 \pm 1.42$} & \multicolumn{2}{|c|}{$1.65 \pm 1.65$} \\
\hline \multirow{3}{*}{$\begin{array}{l}\text { Exponential } \\
\text { phase }(6 \mathrm{~h})\end{array}$} & SNP & \multirow{2}{*}{\multicolumn{2}{|c|}{$1.33 \pm 1.16$}} & \multicolumn{2}{|c|}{$1.47 \pm 0.78^{*}$} \\
\hline & $\mathrm{PFo}$ & $2.00 \pm 1.84^{* *}$ & & \multicolumn{2}{|c|}{$2.07 \pm 1.48^{* *}$} \\
\hline & PFi & \multicolumn{2}{|c|}{$2.17 \pm 1.54^{* *}$} & \multicolumn{2}{|c|}{$2.10 \pm 0.95^{* *}$} \\
\hline \multirow{3}{*}{$\begin{array}{l}\text { Stationary } \\
\text { phase }(24 \text { h) }\end{array}$} & SNP & \multicolumn{2}{|c|}{$2.62 \pm 1.06^{* *}$} & \multicolumn{2}{|c|}{$2.10 \pm 1.60$} \\
\hline & PFo & \multicolumn{2}{|c|}{$2.02 \pm 0.72^{*}$} & \multicolumn{2}{|c|}{$2.45 \pm 1.86^{*}$} \\
\hline & PFi & \multicolumn{2}{|c|}{$1.33 \pm 0.27^{*}$} & \multicolumn{2}{|c|}{$1.93 \pm 1.31$} \\
\hline \multirow{3}{*}{$\begin{array}{l}\text { "Modified" } \\
\text { Exponential } \\
\text { phase }(6 \mathrm{~h})\end{array}$} & SNP & \multicolumn{2}{|c|}{$0.92 \pm 0.73$} & \multicolumn{2}{|c|}{$1.63 \pm 0.75^{* *}$} \\
\hline & PFo & \multicolumn{2}{|c|}{$1.00 \pm 0.76$} & \multicolumn{2}{|c|}{$1.51 \pm 0.66^{* *}$} \\
\hline & PFi & \multicolumn{2}{|c|}{$0.92 \pm 0.68$} & \multicolumn{2}{|c|}{$1.16 \pm 0.70$} \\
\hline
\end{tabular}

"Ratio" is defined as the average HP isozyme concentration of the treated $E$. coli divided by the average HP isozyme concentration of untreated $E$. coli under identical conditions. Significance at $90\left(^{*}\right)$ and $95\left(^{* *}\right)$ percent confidence was determined through Student's T-test and pooled standard deviation. (a)

$1 \mathrm{~h}$
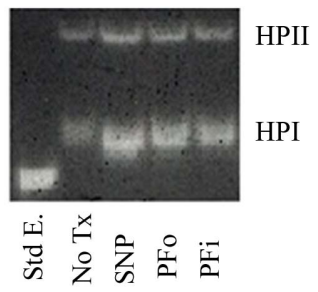

(c)

$24 \mathrm{~h}$

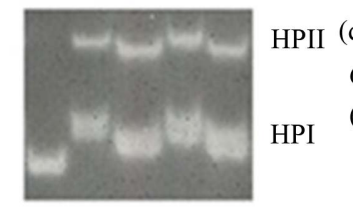

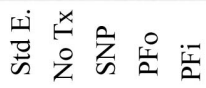

(b)

$6 \mathrm{~h}$

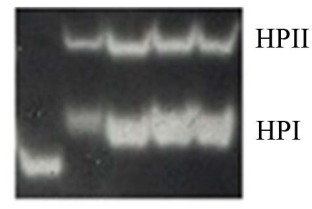

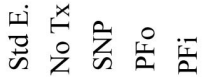

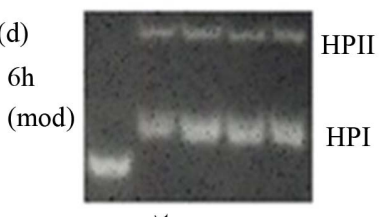

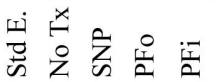

Figure 4. E. coli HP expression profiles, presented as activity bands on non-denaturing polyacrylamide gels. E. coli originating from overnight cultures were incubated as described (see "Methods") in the presence of $1.00 \mathrm{mM}$ sodium nitroprusside (SNP), $1.00 \mathrm{mM}$ potassium ferrocyanide (PFo), or $1.00 \mathrm{mM}$ potassium ferricyanide (PFi), then harvested after 1, 6, or $24 \mathrm{~h}$ of incubation (a-c). "6 h (mod)" are E. coli that were harvested after $6 \mathrm{~h}$ of incubation, but only received $1 \mathrm{~h}$ of exposure to a treatment (addition at $5 \mathrm{~h}$ point) (D). $20 \mu \mathrm{L}$ of $0.6 \mathrm{mg} / \mathrm{ml}$ crude protein extract were loaded into each well, and electrophoresed and stained for HP activity as described (see "Methods"). "Std E." is $10 \mathrm{ng}$ of Corynebacterium glutamicum catalase, used as a positive standard. Under present conditions, E. coli HPII migrates slowest (top bands), and HPI fastest (bottom bands) [73]. Gels are representative of at least three independent trials.

significance even at $\mathrm{P} \leq 0.10$. Heightened HP activity associated with iron treatments was often significant during logarithmic and stationary phases of growth. Unlike SOD, no redox-specific behaviour was observed as PFo and PFi had approximately similar effects on both isozymes of HP. This is evident by the comparatively similar HP activity ratios produced by PFo and PFi throughout all growth phases (Table 2).

Remarkably, "modified" exponential phase trials analogous to those of SOD also revealed phase-dependent enzyme expression, but not in a manner anticipated. Both lag and exponential phase trials displayed conspicuous up-regulation of HP in response to iron challenge. However, "modified" exponential phase trials (exposed to oxidants for only $1 \mathrm{~h}$ ) displayed HPII activity ratios at or near 1, and SNP and PFo induced HPI expression at a level that barely achieved statistical significance These results suggest that the duration of exposure to these oxidants is a significant determining factor in HP expression during exponential phase.

\section{DISCUSSION}

\subsection{SNP Has a Unique Oxidative Impact on E. coli}

Though iron is present in PFi, PFo, and SNP, only the 
NO-releasing SNP slowed exponential phase E. coli and prematurely arrested E. coli at a lower optical density at stationary phase (Figure 1). These results suggest that $\mathrm{NO}$, or NO combined with iron, imparts unique oxidative consequences on $E$. coli. Nitric oxide is a free radical that can directly inflict oxidative damage or combine with other compounds to form powerful oxidative agents [56-58]. Peroxynitrite, the product of the reaction between NO and superoxide, is one such agent that would be rapidly formed in a NO-flooded environment [59]. Peroxynitrite can self-generate by inhibiting electron transport chain complexes, arresting electron flow; and consequently, allow nearby oxygen molecules to steal electrons. Superoxide radicals adventitiously formed then bind with NO to form more peroxynitrite [57]. Considering that exponential phase is characterized by maximal metabolic activity and that the exhaustion of metabolic substrates defines stationary phase, it is plausible that the slowed growth and premature plateau associated with SNP treatment is the consequence of peroxynitrite-associated metabolic waste. This is by no means the only possible explanation.

\subsection{SOD Expression is Immutable to Iron Challenge during Lag Phase}

Exponential phase E. coli exposed to SNP and PFo for $6 \mathrm{~h}$ was determined to have reduced MnSOD expression and enhanced FeSOD expression as compared to untreated cultures, albeit with some statistical uncertainty (Figure 2; Table 1). We have previously suggested that such $\mathrm{Fe}(\mathrm{II})$-associated changes should be mediated by the activation of Fur [47]. The present observations are consistent with studies demonstrating changes in FeSOD and MnSOD activities in iron-replete and iron-deplete conditions in a manner implicating Fur regulation [54, 60]. The dichotomous expression of SOD isozymes was not observed during lag phase (Figure 2; Table 1), and this was accompanied by the observation of dramatically higher MnSOD expression during lag phase as compared to later growth phases (Figure 3). SOD recalcitrance was a puzzling observation considering that iron influx occurs during lag phase [61]; and therefore, dramatic changes in isozyme activities should have been produced through Fur induction. Several studies have suggested that the intracellular concentration of the Fur protein is significantly lower during lag phase of microbial replication. During lag phase there is an impressive influx of iron (and other metals) that would be best mediated by low Fur levels [61]. Fur has been observed to activate the transcription of HPI and HPII, evident by the near absence of both isozymes in mutant $E$. coli lacking Fur ( $\Delta$ fur); however, expression differences between mutant and wildtype strains were only plainly evident after lag phase had ended [23]. This latter observation is consistent with our own observations such that iron challenge may only induce changes in SOD expression once lag phase has ended. Furthermore, E. coli $\Delta$ fur mutants and E. coli incubated in iron-deplete conditions show heightened MnSOD activity akin to what is presently observed in our lag phase cultures $[18,23,54]$. Heightened MnSOD expression is consistent with our hypothesis that Fur activity is low during lag phase because MnSOD would be logically de-repressed at this time. Therefore, based on these present observations and those of other researchers, we hypothesize that heightened SOD expression, and recalcitrant SOD expression, during lag phase are the result of low intracellular levels of the Fur protein. To our best knowledge, no study of the in vivo concentration of the Fur regulator throughout the microbial growth phases has ever been conducted. Such a study would be fortuitous for evaluating this hypothesis, but such an experiment would be beyond the scope of the present research. It should be noted that MnSOD would still be inducible during this phase through other stimulants such as superoxide [62].

\subsection{HP Expression Patterns Suggest Underlying Metabolic Factors Determining HP Activity}

As iron (II)-activated Fur up-regulates HP expression $[21,23]$, we expected to see redox-specific changes in HP activity akin to what was observed in SOD expression profiles. It was instead observed that both PFo and PFi increased HP activity with approximately similar activity ratios throughout all growth phases (Figure 4; Table 2). The unique regulatory role of glutathione in prokaryotes provides a plausible explanation. Though glutathione is commonly known as an endogenous reducing agent, forming disulfide bridges that are then recycled by glutathione reductase and NADPH, in prokaryotic cells glutathione controls the expression of HP [63]. E. coli cells treated with ferricyanide have been observed to increase HPI activity, and glutathione has been implicated in this up-regulation [63,64]. As PFo may induce higher HPI expression through Fur, and not through other HP regulators such as the protein products of oxyR or rpoS [21], both iron (II) and iron (III) are capable of up-regulating HP activity by independent means. Heightened HP activity in response to both PFo and PFi may therefore be the consequence of the involvement of both Fur and glutathione, and of the oxidative impact inflicted by these treatments necessitating such a robust anti-oxidative response.

Both exponential and stationary phase cultures presented substantial increases in HP activity in response to iron treatment (Table 2; Figures 4(b) and (c)). Exponen- 
tial phase is the most metabolically active phase, producing ten times more hydrogen peroxide than any other phase [65-67]. To maintain homeostasis, cells respond to these generated oxidative and/or free radical stresses by dramatically up-regulating HP activity [65-67]. Through the catalysis of radicals by the Fenton reaction, the oxidative impact of iron would be greatest at this time. It should not be surprising to see such a robust response to these iron treatments during exponential phase (Figure 4(b); Table 2). Heightened HP activity during stationary phase is also most probably the consequence of aerobic metabolism; and specifically, the acidification of the media. We have observed that the $\mathrm{pH}$ of these glucose-based cultures decreased over time (data not shown), consistent with other $\mathrm{pH}$ studies [26]. The accumulation of weak acids in stationary phase is complacent in the up-regulation of HP $[34,68]$. Furthermore, Fenton's own studies [69] and subsequent research by Walling [70] revealed that in mildly acidic environments, hydrogen peroxide may regenerate iron (II) from iron (III), generating peroxyl radicals and hydrogen cations (Eq.4):

$$
\mathrm{Fe}^{3+}+\mathrm{H}_{2} \mathrm{O}_{2} \longrightarrow \mathrm{Fe}^{2+}+\mathrm{HOO} \cdot+\mathrm{H}^{+}
$$

The low $\mathrm{pH}$ in stationary phase media would therefore exacerbate the oxidative impact of iron and would further explain why the effects of PFi and PFo on HP expression are similar.

\subsection{Unexpected Findings and Study Limitations}

As studies using $E$. coli lacking Fur $(\Delta$ fur) have shown [23], Fur is necessary for robust HP activity during lag phase. In accordance with our hypothesis of low Fur activity at this time, we had expected HP activity to be quite low compared to other phases at this time and that, like SOD, HP expression should be unresponsive to iron challenge. HP expression was indeed found to be much lower during lag phase than, say, exponential phase (data not shown); however, iron treatment did produce activity ratios deviating considerably from the expected value of 1 , with much statistical uncertainty (Table 2 ). We have had some difficulty obtaining consistent data for HP during lag phase, and the error margins were quite large. We have previously observed in unpublished work that HP expression during lag phase is highly malleable to specific incubatory conditions. For example, the age of the over-night starter culture used to inoculate fresh media for incubation is one such consideration: If it is old (e.g., one week) it decreases MnSOD activity by two thirds and doubles HPII activity, as compared to fresh starter culture. Harvest time within the lag period is another consideration: HPI activity is 10 -fold higher 40 minutes post-inoculation than at 60 or 80 minutes postinoculation. This heightened activity may possibly be an artifact from the starter culture as it exits stationary phase to renew replication in a nutrient-replete environment. If so, this would implicate this 40 to 60 minute interval as a transitional period for HP activity, and any experimental work during this time is bound to produce much variance in data. Future studies harvesting at 80 minutes under identical conditions may remove this source of error. Temperature is yet another consideration: To illustrate the effects of extreme environmental change, we have observed that when a starter culture is cooled to $4^{\circ} \mathrm{C}$ then added to fresh media set at $37^{\circ} \mathrm{C}$, both HP and SOD expression are affected as compared to starter cultures kept at $37^{\circ} \mathrm{C}$ throughout. These observations may be of interest in elucidating the antioxidant profile during this most poorly understood phase of microbial replication. We did not encounter data consistency issues when assaying SOD activity during lag phase, which instead showed remarkably consistent data that proved useful for formulating the present hypothesis.

Each enzyme activity ratio is the mean value of a treated condition divided by the mean value of the corresponding untreated standard. Hence, the standard deviation for each activity ratio is the product of the error of the treated data set and the error of the untreated data set. This approach will inevitably produce considerably large error margins in the final activity ratios given smaller error margins in either data set. We used the present methodology because it has previously shown to be a simple and successful means of investigating antioxidant expression profiles in microbial culture [47]. Although we are confident that SOD expression is both heightened and unresponsive to iron challenge during lag phase, the considerable error margins produced by the methodological approach in many of the treatments and growth phases stresses caution in interpretation. Some changes in SOD or HP activity induced by iron treatments proved statistically significant, but others did not. Alternative experimental approaches could be useful for supporting the present observations and further elucidating the role of Fur in regulating antioxidant enzymes in response to iron threats.

\section{ACKNOWLEDGEMENTS}

This work was supported by the following grants which are hereby gratefully acknowledged: Research Corporation Cottrell College Science Award \#5926 [Research Corporation, 4703 E. Camp Lowell Drive, Suite 201, Tucson, AZ 85712, USA] to MOE; two University of Winnipeg (UofW) Major Research Grants \#7437 and \#7671 to MOE; and a Special Grant from the UofW Research and Graduate Studies Office to Dr. Desiree Vanderwel (Chemistry Dept. UofW) and MOE. We are also grateful to Mr. Ramin Vakili (Chemistry Dept., UofW) and Dr. Jamie Galka (Chemistry Dept., UofW) for valuable technical assistance. An NSERC PGS-M (425305-2012) awarded to RLB is also gratefully acknowledged. We also thank two anonymous reviewers for their insightful 
critiques on previous drafts of the manuscript.

\section{REFERENCES}

[1] Benov, L.T. (2001) How superoxide radical damages the cell. Protoplasma, 217, 33-36. http://dx.doi.org/10.1007/BF01289410

[2] Cerutti, P.A. (1985) Prooxidant states and tumor promotion. Science, 227, 375-381. http://dx.doi.org/10.1126/science.2981433

[3] Halliwell, B. (1987) Oxidants and human disease: Some new concepts. Journal of the Federation of American Societies for Experimental Biology, 1, 358-364.

[4] Harman, D. (1991) The aging process: Major risk factor for disease and death. Proceedings of the National Academy of Sciences of the United States of America, 88, 5360-5363. http://dx.doi.org/10.1073/pnas.88.12.5360

[5] González-Flecha, B., Cutrin, J.C. and Boveris, A. (1993) Time course and mechanism of oxidative stress and tissue damage in rat liver subjected to in vivo ischemia-reperfusion. Journal of Clinical Investigations, 91, 456-464. http://dx.doi.org/10.1172/JCI116223

[6] Luft, R. (1994) The development of mitochondrial medicine. Proceedings of the National Academy of Sciences of the United States of America, 91, 8731-8738. http://dx.doi.org/10.1073/pnas.91.19.8731

[7] Yan, S.D., Chen, X., Schmidt, A.M., Brett, J., Godman, G., Zou, Y.S., et al. (1994) Glycated tau protein in Alzheimer disease: A mechanism for induction of oxidant stress. Proceedings of the National Academy of Sciences of the United States of America, 91, 7787-7791.

http://dx.doi.org/10.1073/pnas.91.16.7787

[8] Muller, F.L., Song, W., Liu, Y., Chaudhuri, A., Pieke-Dahl, S., Strong, R., et al. (2006) Absence of CuZn superoxide dismutase leads to elevated oxidative stress and acceleration of age-dependent skeletal muscle atrophy. Free Radical Biology and Medicine, 40, 1993-2004. http://dx.doi.org/10.1016/j.freeradbiomed.2006.01.036

[9] Halliwell, B. and Gutteridge, J.M. (1984) Oxygen toxicity, oxygen radicals, transition metals and disease. Biochemistry Journal, 219, 1-14.

[10] Halliwell, B. and Gutteridge, J.M. (1997) Lipid peroxidation in brain homogenates: The role of iron and hydroxyl radicals. Journal of Neurochemistry, 69, 1330-1331. http://dx.doi.org/10.1046/j.1471-4159.1997.69031330.x

[11] Bagg, A. and Neilands, J.B. (1987) Molecular mechanism of regulation of siderophore-mediated iron assimilation. Microbiology Reviews, 51, 509-518.

[12] Hubbard, J.A.M., Lewandowska, K.B., Hughes, M.N. and Poole, R.K. (1986) Effects of iron-limitation of Escherichia coli on growth, the respiratory chains and gallium uptake. Archives of Microbiology, 146, 80-86. http://dx.doi.org/10.1007/BF00690163

[13] Bagg, A. and Neilands, J.B. (1987) Ferric uptake regulation acts as a repressor, employing iron (II) as a cofactor to bind the operator of an iron transport operon in Escherichia coli. Biochemistry, 26, 5471-5477.

http://dx.doi.org/10.1021/bi00391a039
[14] Escolar, L., Pérez-Martin, J. and De Lorenzo, V. (1999) Opening the iron box: Transcriptional metalloregulation by the Fur protein. Journal of Bacteriology, 181, 62236229.

[15] Hantke, K. (2001) Iron and metal regulation in bacteria. Current Opinion in Microbiology, 4, 172-177. http://dx.doi.org/10.1016/S1369-5274(00)00184-3

[16] Tardat, B. and Touati, D. (1991) Two global regulators repress the anaerobic expression of MnSOD in E. coli: Fur (ferric uptake regulation) and Arc (aerobic respiration control). Molecular Microbiology, 5, 455-465. http://dx.doi.org/10.1111/j.1365-2958.1991.tb02129.x

[17] Tardat, B. and Touati, D. (1993) Iron and oxygen regulation of Escherichia coli MnSOD expression: Competition between the global regulators Fur and ArcA for binding to DNA. Molecular Microbiology, 9, 53-63. http://dx.doi.org/10.1111/j.1365-2958.1993.tb01668.x

[18] Hassett, D.J., Sokol, P.A., Howell, M.L., Ma, J.F., Schweizer, H.T., Ochsner, U., et al. (1996) Ferric uptake regulator (Fur) mutants of Pseudomonas aeruginosa demonstrate defective siderophore-mediated iron uptake, altered aerobic growth, and decreased superoxide dismutase and catalase activities. Journal of Bacteriology, 178, 3996-4003.

[19] Dubrac, S. and Touati, D. (2000) Fur positive regulation of iron superoxide dismutase in Escherichia coli: Functional analysis of the sodB promoter. Journal of Bacteriology, 182, 3802-3808. http://dx.doi.org/10.1128/JB.182.13.3802-3808.2000

[20] Dubrac, S. and Touati, D. (2002) Fur-mediated transcriptional and post-transcriptional regulation of FeSOD expression in Escherichia coli. Microbiology, 148, 147-156.

[21] Zaid, T., Sukumaran, T., Srikumar, N. and Benov, L. (2003) Growth of Escherichia coli in iron-enriched medium increases HPI catalase activity. Journal of Biochemistry and Molecular Biology, 36, 608-610. http://dx.doi.org/10.5483/BMBRep.2003.36.6.608

[22] Benov, L. and Sequeira, F. (2003) Escherichia coli $\Delta$ fur mutant displays low HPII catalase activity in stationary phase. Redox Report, 8, 379-383. http://dx.doi.org/10.1179/135100003225003357

[23] Hoerter, J.D., Arnold, A.A., Ward, C.S., Sauer, M., Johnson, S., Fleming, T., et al. (2005) Reduced hydroperoxidase (HPI and HPII) activity in the delta fur mutant contributes to increased sensitivity to UVA radiation in Escherichia coli. Journal of Photochemistry and Photobiology $B, 79,151-157$. http://dx.doi.org/10.1016/j.jphotobiol.2005.01.003

[24] Schellhorn, H.E. and Hassan, H.M. (1988) Response of hydroperoxidase and superoxide dismutase deficient mutants of Escherichia coli K-12 to oxidative stress. Canadian Journal of Microbiology, 34, 1171-1176. http://dx.doi.org/10.1139/m88-206

[25] Park. S., You, X. and Imlay, J.A. (2005) Substantial DNA damage from submicromolar intracellular hydrogen peroxide detected in Hpx- mutants of Escherichia coli. Proceedings of the National Academy of Sciences of the United States of America, 102, 9317-9322.

http://dx.doi.org/10.1073/pnas.0502051102 
[26] Hassan, H.M. and Fridovich, I. (1977) Regulation of superoxide dismutase synthesis in Escherichia coli: Glucose effect. Journal of Bacteriology, 132, 505-510.

[27] Korshunov, S. and Imlay, J.A. (2006) Detection and quantification of superoxide formed within the periplasm of Escherichia coli. Journal of Bacteriology, 188, 63266334. http://dx.doi.org/10.1128/JB.00554-06

[28] Hopkin, K.A., Papazian, M.A. and Steinman, H.M. (1992) Functional differences between manganese and iron superoxide dismutases in Escherichia coli K-12. Journal of Bacteriology, 267, 24253-24258.

[29] Compan, I. and Touati, D. (1993) Interaction of six global transcription regulators in expression of manganese superoxide dismutase in Escherichia coli K-12. Journal of Bacteriology, 175, 1687-1696.

[30] Triggs-Raine, B.L., Doble, B.W., Mulvey, M.R., Sorby, P.A. and Loewen, P.C. (1988) Nucleotide sequence of katG, encoding catalase HPI of Escherichia coli. Journal of Bacteriology, 170, 4415-4419.

[31] von Ossowski, I., Mulvey, M.R., Leco, P.A., Borys, A. and Loewen, P.C. (1991) Nucleotide sequence of Escherichia coli katE, which encodes catalase HPII. Journal of Bacteriology, 173, 514-520.

[32] Schellhorn, H.E. (1995) Regulation of hydroperoxidase (catalase) expression in Escherichia coli. Federation of European Microbiological Societies: Microbiology Letters, 131, 113-119. http://dx.doi.org/10.1111/j.1574-6968.1995.tb07764.x

[33] Mulvey, M.R., Switala, J., Borys, A. and Loewen, P.C. (1990) Regulation of transcription of katE and katF in Escherichia coli. Journal of Bacteriology, 172, 67136720.

[34] Schellhorn, H.E. and Stones, V.L. (1992) Regulation of katF and katE in Escherichia coli K-12 by weak acids. Journal of Bacteriology, 174, 4769-4776.

[35] Eisenstark, A., Calcutt, M.J., Becker-Hapak, M. and Ivanova, A. (1996) Role of Escherichia coli rpoS and associated genes in defense against oxidative damage. Free Radicals in Biology and Medicine, 21, 975-993. http://dx.doi.org/10.1016/S0891-5849(96)00154-2

[36] Loewen, P.C. and Hengge-Aronis, R. (1994) The role of the sigma factor sigma $\mathrm{S}$ (KatF) in bacterial global regulation. Annual Review of Microbiology, 48, 53-80. http://dx.doi.org/10.1146/annurev.mi.48.100194.000413

[37] Ivanova, A., Miller, C., Glinsky, G. and Eisenstark, A. (1994) Role of rpoS (katF) in oxyR-independent regulation of hydroperoxidase I in Escherichia coli. Molecular Microbiology, 12, 571-578. http://dx.doi.org/10.1111/j.1365-2958.1994.tb01043.x

[38] Panacek, E.A., Bednarczyk, E.M., Dunbar, L.M., Foulke, G.E. and Holcslaw, T.L. (1995) Randomized, prospective trial of fenoldopam vs sodium nitroprusside in the treatment of acute severe hypertension. Fenoldopam Study Group. Academic Emergency Medicine, 2, 959-965. http://dx.doi.org/10.1111/j.1553-2712.1995.tb03122.x

[39] Pathak, A., Mathuriya, S.N., Khandelwal, N. and Verma, K. (2003) Intermittent low dose intrathecal sodium nitroprusside therapy for treatment of symptomatic aneurismal SAH- induced vasospasm. British Journal of Neurosurgery, 17, 306-310.

http://dx.doi.org/10.1080/02688690310001601180

[40] Mullens, W., Abrahams, Z., Francis, G.S., Skouri, H.N., Starling, R.C., Young, J.B., et al. (2008) Sodium nitroprusside for advanced low-output heart failure. Journal of the American College of Cardiology, 52, 200-207. http://dx.doi.org/10.1016/j.jacc.2008.02.083

[41] Chantler, P.D., Nussbacher, A., Gerstenblith, G., Schulman, S.P., Becker, L.C., Ferrucci, L., Fleg, J.L., Lakatta, E.G. and Najjar, S.S. (2011) Abnormalities in arterial-ventricular coupling in older healthy persons are attenuated by sodium nitroprusside. American Journal of Physiology: Heart and Circulatory Physiology, 300, H1914- H1922. http://dx.doi.org/10.1152/ajpheart.01048.2010

[42] Yannopoulos, D., Matsuura, T., Schultz, J., Rudser, K., Halperin, H.R. and Lurie, K.G. (2011) Sodium nitrorprusside enhanced cardiopulmonary resuscitation improves survival with good neurological function in a porcine model of prolonged cardiac arrest. Critical Care Medicine, 39, 1269-1274.

[43] Grossi, L. and D'Angelo, S. (2005) Sodium nitroprusside: Mechanism of NO release mediated by sulfhydryl-containing molecules. Journal of Medicinal Chemistry, 48, 2622-2626. http://dx.doi.org/10.1021/jm049857n

[44] D'Autreaux, B., Touati, D., Bersch, B., Latour, J.M. and Michaud-Soret, I. (2002) Direct inhibition by nitric oxide of the transcriptional ferric uptake regulation protein via nitrosylation of the iron. Proceedings of the National Academy of Sciences of the United States of America, 99, 16619-16624. http://dx.doi.org/10.1073/pnas.252591299

[45] Foster, M.W., McMahon, T.J. and Stamler, J.S. (2003) SNitrosylation in health and disease. Trends in Molecular Medicine, 9, 160-168. http://dx.doi.org/10.1016/S1471-4914(03)00028-5

[46] Szacilowski, K., Chmura, A. and Stasicka, Z. (2005) Interplay between iron complexes, nitric oxide and sulfur ligands: Structure, (photo)reactivity and biological importance. Coordination Chemistry Reviews, 249, 2408-2436. http://dx.doi.org/10.1016/j.ccr.2005.03.021

[47] Bertrand, R., Danielson, D., Gong, V., Olynik, B. and Eze, M.O. (2012) Sodium nitroprusside may modulate Escherichia coli antioxidant enzyme expression by interacting with the ferric uptake regulator. Medical Hypotheses, 78, 130133. http://dx.doi.org/10.1016/j.mehy.2011.10.007

[48] Nunoshiba, T., De Rojas-Walker, T., Wishnok, J.S., Tannenbaum, S.R. and Demple, B. (1993) Activation by nitric oxide of an oxidative-stress response that defends Escherichia coli against activated macrophages. Proceedings of the National Academy of Sciences of the United States of America, 90, 9993-9997. http://dx.doi.org/10.1073/pnas.90.21.9993

[49] Vasil'eva, S.V., Stupakova, M.V., Lobysheva, I.I., Mikoyan, V.D. and Vanin, E.F. (2001) Activation of the Escherichia coli SoxRS-Regulon by nitric oxide and its physiological donors. Biochemistry, 66, 984-988.

[50] Niederhoffer, E.C., Naranjo, C.M., Bradley, K.L. and Fee, J.A. (1990) Control of Escherichia coli superoxide dismutase (sodA and sodB) genes by the ferric uptake regu- 
lation (fur) locus. Journal of Bacteriology, 172, 1930-1938.

[51] Massé, E. and Gottesman, S. (2002) A small RNA regulates the expression of genes involved in iron metabolism in Escherichia coli. Proceedings of the National Academy of Sciences of the United States of America, 99, 46204625. http://dx.doi.org/10.1073/pnas.032066599

[52] Bradford, M.M. (1976) A rapid and sensitive method for the quantitation of microgram quantities of protein utilizing the principle of protein-dye binding. Analytical Biochemistry, 72, 248-254.

http://dx.doi.org/10.1016/0003-2697(76)90527-3

[53] Beauchamp, C. and Fridovich, I. (1971) Superoxide dismutase: Improved assays and an assay applicable to acrylamide gels. Analytical Biochemistry, 44, 276-287. http://dx.doi.org/10.1016/0003-2697(71)90370-8

[54] Britigan, B.E., Miller, R.A., Hassett, D.J., Pfaller, M.A., McCormick, M.L. and Rasmussen, G.T. (2001) Antioxidant enzyme expression in clinical isolates of Pseudomonas aeruginosa: Identification of an aytypical form of manganese superoxide dismutase. Infection and Immunity, 69, 7396-7401. http://dx.doi.org/10.1128/IAI.69.12.7396-7401.2001

[55] Woodbury, W., Spencer, A.K. and Stahman, M.A. (1971) An improved procedure using ferricyanide for detecting catalase isozymes. Analytical Biochemistry, 44, 301-305. http://dx.doi.org/10.1016/0003-2697(71)90375-7

[56] Zaki, M.H., Akuta, T. and Akaike, T. (2005) Nitric oxide-induced nitrative stress involved in microbial pathogenesis. Journal of Pharmacological Sciences, 98, 117129. http://dx.doi.org/10.1254/jphs.CRJ05004X

[57] Pacher, P., Beckman, J.S. and Liaudet, L. (2007) Nitric oxide and peroxynitrite in health and disease. Physiological Reviews, 87, 315-424.

http://dx.doi.org/10.1152/physrev.00029.2006

[58] Qu, W., Zhou, Y.B., Shao, C.H., Sun, Y.D., Zhang, Q.Y., Chen, C.Y. and Jia, J.H. (2009) Helicobacter pylori proteins response to nitric oxide stress. Journal of Microbiology, 47, 486-493. http://dx.doi.org/10.1007/s12275-008-0266-0

[59] Huie, R.E. and Padmaja, S. (1993) The reaction rate of nitric oxide with superoxide. Free Radical Research, 18, 195-199. http://dx.doi.org/10.3109/10715769309145868

[60] Privalle, C.T. and Fridovich, I. (1993) Iron specificity of the Fur-dependent regulation of the biosynthesis of the manganese-containing superoxide dismutase in Escherichia coli. Journal of Biological Chemistry, 268, 5178-5181.

[61] Rolfe, M.D., Rice, C.J., Lucchini, S., Pin, C., Thompson, A., Cameron, A.D.S., et al. (2012) Lag phase is a distinct growth phase that prepares bacteria for exponential growth and involves transient metal accumulation. Journal of Bacteriology, 194, 686-701.

http://dx.doi.org/10.1128/JB.06112-11
[62] Kim, J.S., Sung, M.H., Kho, D.H. and Lee, J.K. (2005) Induction of managanese-containing superoxide dismutase is required for acid tolerance in Vibrio vulnificus. Journal of Bacteriology, 187, 5984-5995. http://dx.doi.org/10.1128/JB.187.17.5984-5995.2005

[63] Oktyabrsky, O.N., Smirnova, G.V. and Muzyka, N.G. (2001) Role of glutathione in regulation of hydroperoxidase I in growing Escherichia coli. Free Radicals in Biology and Medicine, 31, 250-255.

http://dx.doi.org/10.1016/S0891-5849(01)00572-X

[64] Smirnova, G.V., Muzyka, N.G., Glukhovchenko, M.N. and Oktyabrsky, O.N. (1997) Effects of penetrating and nonpenetrating oxidants on Escherichia coli. Biochemistry, 62, 480-484.

[65] González-Flecha, B. and Demple, B. (1995) Metabolic sources of hydrogen peroxide in aerobically growing Escherichia coli. Journal of Biological Chemistry, 270, 13681-13687. http://dx.doi.org/10.1074/jbc.270.23.13681

[66] González-Flecha, B. and Demple, B. (1997) Homeostatic regulation of intracellular hydrogen peroxide concentration in aerobically growing Escherichia coli. Journal of Bacteriology, 179, 382-388.

[67] González-Flecha, B. and Demple, B. (1997) Transcriptional regulation of the Escherichia coli oxyR gene as a function of cell growth. Journal of Bacteriology, 179, 61816186.

[68] Mukhopadhyay, S. and Schellhorn, H.E. (1994) Induction of Escherichia coli hydroperoxidase I by acetate and other weak acids. Journal of Bacteriology, 176, 2300-2307.

[69] Fenton, H.J.H. (1894) Oxidation of tartaric acid in presence of iron. Journal of the Chemical Society, Transactions, 65, 899-910.

http://dx.doi.org/10.1039/ct8946500899

[70] Walling, C. (1975) Fenton's reagent revisited. Accounts of Chemical Research, 8, 125-131. http://dx.doi.org/10.1021/ar50088a003

[71] Benov, L.T. and Fridovich, I. (1994) Escherichia coli expresses a copper- and zinc-containing superoxide dismutase. The Journal of Biological Chemistry, 269, 2531025314.

[72] Bereswill, S., Neurner, O., Strobel, S. and Kist, M. (2000) Identification and molecular analysis of superoxide dismutase isoforms in Heliobacter pylori. Federation of European Microbiological Societies: Microbiology Letters, 183, 241-245.

http://dx.doi.org/10.1111/j.1574-6968.2000.tb08965.x

[73] Loewen, P.C., Triggs, B.L., George, C.S. and Hrabarchuk, B.E. (1985) Genetic mapping of katG, a locus that affects synthesis of the bifunctional catalase-peroxidase hydroperoxidase I in Escherichia coli. Journal of Bacteriology, 162, 661-667. 